BGU-10-92

hep-th/9302081

\title{
Threefold Family of Charged Spin-1/2 Dirac Bubbles
}

\author{
Aharon Davidson* and Uzi Paz** \\ Physics Department \\ Ben-Gurion University of the Negev \\ Beer-Sheva 84105, Israel
}

(Phys. Lett. B, in press)

\begin{abstract}
We extend Dirac's 'extensible model of the electron' to include spin and family. $U(1)_{\text {e.m. }}$ charge conservation on the bubble is translated into a secondary $U(1)_{\mathrm{g}}$ world-manifold gauge principle. Reflecting the secondary magnetic monopole configuration on spatial $S^{2}$, the harmonic excitations may furnish half integer $S U(2)_{\text {spin }} \otimes U(1)_{\mathrm{g}}$ representations. Our spin-1/2 'electron' is described by four world-manifold scalar fields. Its three varieties are associated with different minima of the $\left(6^{\text {th }}\right.$-order $)$ surface-tension scalar potential.
\end{abstract}

*E-mail: davidson@bguvms

${ }^{* *}$ E-mail: uzipaz@bgumail.bgu.ac.il 


\section{Introduction}

The long-lived idea of a finite size elementary particle is as appealing as ever. Adopting Dirac's viewpoint, such an idea may be "the most natural concept that makes the total energy of the Coulomb field of the electron finite". Experimentally, however, up to a scale of $10^{-16} \mathrm{~cm}$, quarks and leptons exhibit a point-like behavior. Nonetheless, their apparently superfluous proliferation (exactly three families) gives us some reason to suspect an internal structure. The finite electron idea has been introduced [1], on a classical level, by Abraham and Lorentz at the dawn of special relativity. Later on, Einstein [2] has examined the possibility that gravitational fields play an essential role in the game. He was even ready to modify his field equations in order to "elaborate a theory which will account for the equilibrium of the electricity constituting the electron". His overall conclusion was, however, that the problem cannot be explained solely by means of a locally varying cosmological constant.

The constitution of the elementary quanta was retackled and sophistically refined by Dirac [3]. He pictured the electron as a breathing bubble in the electromagnetic field, with no constraints fixing its size and shape. An effective surface tension was postulated in order to prevent the electron from flying apart under the Coulomb repulsion of its surface charge. The naive, yet pedagogical 'extensible model of the electron' was an attempt to deal with Rabi's immortal question 'who ordered the muon?'. The major drawback of the model was its impotency to incorporate spin. Nowadays, being exposed to modern theories such as grand-unification, supergravity, superstrings, and hypothetical quantum gravity, claiming to take us beyond the standard Glashow-Weinberg-Salam electronuclear model, it is quite ironic that one still lacks a decent clue regarding the family puzzle and the structure of the Fermi mass matrix. Imaginative preon models [4], accompanied by hyper-color theories [5] especially designed to handle QCD-like quark/lepton compositeness, have not shed light on these problems. The intuitive approach advertised by Dirac, which in our opinion has not been examined to its full depth, may eventually turn out to be a step in the right direction.

The physics that takes us beyond the Dirac limit is formulated here in terms of a world-manifold local gauge theory. Invoking various field and group theoretical ideas in $2+1$ dimensions (i.e. on the bubble), while fully respecting $U(1)_{\text {e.m. }}$ gauge invariance in $3+1$ dimensions (i.e. surrounding the bubble), we construct a selfconsistent Lagrangian formalism which, up to weak interactions (to be hopefully incorporated in future stage), fairly represents a finite-size electron-like extended object. Our work attempts to (i) account for the spontaneous (rather than explicit) generation of spin, (ii) construct an explicit spin-1/2 static soliton configuration in space-time, (iii) offer a possible solution to the family puzzle, and (iv) re-examine the problem of mass. The formalism is $\mathrm{P} / \mathrm{C} / \mathrm{T}$ conserving, and has nothing to do with the Chern-Simons theory, the popular source of fractional statistics [6] in $2+1$ 
dimensions. Due to length limitations, however, we skip some lengthy calculations (to be published elsewhere [7]). For the sake of clarity, we proceed in steps towards eqs. (18), (20).

\section{2. $\mathrm{U}(1)_{\mathrm{e} . \mathrm{m} .}$ Gauge Invariance}

Our starting point is conventional. We work in a flat $3+1$ dimensional background with metric $G_{\mu \nu}(x)$. The bubble's $(2+1$ dimensional) world-manifold is parametrized by $x^{\mu}\left(y^{\alpha}\right)$, so that its induced metric and projective electromagnetic field take the form

$$
g_{\alpha \beta} \equiv G_{\mu \nu} x^{\mu}{ }_{\alpha} x^{\nu}{ }_{, \beta} \quad, \quad A_{\alpha} \equiv A_{\mu} x^{\mu},_{\alpha},
$$

respectively. We are after a self-consistent $A^{\mu}(x)$ which arises from some given (to be dynamical) charge/current surface distribution $Q^{\alpha}(y)$. The interaction of the electromagnetic field with our bubble is conventionally described by the $U(1)_{\text {e.m. }}$. invariant action

$$
S^{(1)}=\int \frac{1}{4} G^{\mu \lambda} G^{\nu \sigma} F_{\mu \nu} F_{\lambda \sigma} \sqrt{-\operatorname{det} G} d^{4} x+\oint Q^{\alpha} A_{\alpha} d^{3} y
$$

Note that it is $q^{\alpha}=\frac{Q^{\alpha}}{\sqrt{-\operatorname{det} g}}$, and not $Q^{\alpha}$, which transforms like a legitimate $2+1$ vector.

A variation with respect to $A_{\mu}(x)$, with the bubble treated as an internal boundary, gives rise to the boundary condition

$$
F^{\mu \nu} n_{\nu}=\frac{Q^{\alpha} x^{\mu}, \alpha}{\sqrt{-\operatorname{det} g}}
$$

for the free Maxwell equations outside the bubble. $n^{\mu}$ denotes here a space-like unit normal vector pointing inwards. For the radially symmetric case, defined by $\{t=\tau$, $r=r(\tau), \theta=\theta, \phi=\phi\}$, the Coulomb potential $A_{t}=\frac{q}{r}$ originates from

$$
Q^{\tau}=q \sin \theta \quad, \quad Q^{\theta}=Q^{\phi}=0 .
$$

Notice that in the general case, $U(1)_{\text {e.m. }}$ gauge invariance is not automatically guarantied. In fact, for $S^{(1)}$ to stay trivial if $A_{\mu}=\partial_{\mu} \Lambda$ (translated into $A_{\alpha}=\partial_{\alpha} \Lambda$ ), $Q^{\alpha}$ better obey $Q^{\alpha},{ }_{\alpha}=0$. For a point particle, a constant $Q^{\tau}$ will do, but for a higherdimensional extended object, such a conservation law requires pre-arrangement. 


\section{A Secondary Gauge Principle}

$Q^{\alpha}$ is locally conserved on the world-manifold if and only if

$$
Q^{\alpha}=\epsilon^{\alpha \beta \gamma} \partial_{\beta} a_{\gamma} .
$$

The fact that $a_{\alpha}(y)$, which has not been explicitly introduced in the Dirac theory, is

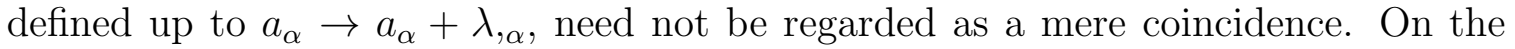
contrary, it is highly suggestive to add the kinetic term [8]

$$
S^{(2)}=\oint \frac{1}{4 m} g^{\alpha \gamma} g^{\beta \delta} f_{\alpha \beta} f_{\gamma \delta} \sqrt{-\operatorname{det} g} d^{3} y,
$$

and treat $a_{\alpha}(y)$ as a canonical world-manifold gauge field. The mass scale $m$ serves here to scale $a_{\alpha}$ to conveniently match the units of $A_{\mu}$.

Spherical symmetry uniquely means [9]

$$
a_{\tau}=a_{\theta}=0 \quad, \quad a_{\phi}=\mathrm{g}(1-\cos \theta),
$$

which is recognized as a non-singular (for a non-collapsing bubble) $2+1$ dimensional magnetic-monopole-like configuration. Notice that $\mathrm{g}$, the secondary magnetic charge, plays the double role of being the primary electric charge $q$ as well. Special attention is devoted to the so-called Dirac pole, the trace of a hypothetical Dirac-string [10]. In the absence of a Chern-Simons term (which directly converts flux points to electric charges) the Dirac pole is not an anyon [6]. Its location at the south pole is just a matter of convenience, nothing but choosing the $z$-axis without spoiling the spherical symmetry. As could have been anticipated, the Dirac-pole turns out to be an artifact only for properly quantized $U(1)_{\mathrm{g}}$ charges, and only then, can be gauged away by means of a secondary Wu-Yang-like construction.

An important observation is that the above monopole solution of $S^{(1)}+S^{(2)}$ stays stable against variations with respect to $a_{\alpha}(y)$, i.e. it automatically satisfies the equation of motion

$$
\left(\frac{1}{m} \sqrt{-\operatorname{det} g} f^{\alpha \beta}\right)_{, \beta}=\frac{1}{2} \epsilon^{\alpha \beta \gamma} F_{\beta \gamma},
$$

for arbitrary g. The general integration of this equation demonstrates how our bubble acts as a superconductor [11]. One can verify that, as far as the real photon is concerned, the combined effect of the two $a_{\alpha}$ terms in the Lagrangian is identical to that of a single mass term $\frac{1}{2} m \sqrt{-\operatorname{det} g} g^{\alpha \beta} A_{\alpha} A_{\beta}$. 


\section{Dynamical Bubble}

Following Dirac, there should be no constraints fixing the size and shape of the bubble. In other words, $x^{\mu}\left(y^{\alpha}\right)$ must serve as a canonical variable. The action integral $\oint L d^{3} y$ is then handled in the standard way, whereas $\int \frac{1}{4} F^{2} d^{4} x$ poses a minor technical problem when noticing, that a variation with respect to $x^{\mu}\left(y^{\alpha}\right)$ changes its internal boundary. Taking into account the later effect, the corresponding equation of motion reads

$$
\frac{\delta L}{\delta x^{\mu}}-\frac{\partial}{\partial y^{\alpha}}\left(\frac{\delta L}{\delta x_{, \alpha}^{\mu}}\right)-\frac{1}{4} \sqrt{-\operatorname{det} g} G^{\nu \sigma} G^{\lambda \xi} F_{\nu \lambda} F_{\sigma \xi} n_{\mu}=0
$$

with the unit normal vector $n^{\mu}$ defined earlier. The last term expresses the back reaction, i.e. the force exerted on the bubble by the surrounding electromagnetic field.

Unfortunately, $S^{(1)}+S^{(2)}$ is not capable of preventing the bubble from flying apart under the Coulomb repulsion. Dirac, not exercising the option of introducing worldmanifold fields, has invoked some effective surface-tension (positive world-manifold cosmological term)

$$
\bar{S}^{(3)}=\oint \Lambda \sqrt{-\operatorname{det} g} d^{3} y .
$$

Such a description, although qualitative, is sufficient for deriving a bounded expression for the total energy. In particular, for the spherically-symmetric case, one obtains

$$
E(r)=\Lambda r^{2}+\frac{\mathrm{g}^{2}}{2 m r^{2}}+\frac{3 q^{2}}{2 r}
$$

where the electromagnetic surface contribution $\frac{q^{2}}{r}$ appears to be twice the electromagnetic volume contribution $\frac{q^{2}}{2 r}$. Discussing geometrical terms, one may wonder whether a curvature term $\tilde{m} \oint R \sqrt{-\operatorname{det} g} d^{3} y$ makes sense as well. We will return to this option later on, when confronting the problem of mass.

\section{Threefold Family Structure}

The most naive interpretation of $\Lambda$ has to do with the vacuum-expectation-value (VEV) of some world-manifold scalar potential. Such a familiar point of view (see e.g. 
inflationary cosmology) has one immediate advantage. Namely, the scalar potential may admit several isolated minima. This may open the door for a finite family structure, establishing a viable alternative to Dirac's radial excitation idea. Our simplest choice is a real, that is, $U(1)_{\mathrm{g}}$-neutral, scalar field, with

$$
S^{(3)}=\oint \sqrt{-\operatorname{det} g}\left(g^{\alpha \beta} \Phi,_{\alpha} \Phi, \beta+V(\Phi)\right) d^{3} y
$$

replacing $\bar{S}^{(3)}$. The reason for choosing a real scalar is first of all technical. Since the covariant and the ordinary derivatives are the one and the same in this case, the $a_{\alpha}$-equation of motion need not be re-examined. A single complex field, on the other hand, would not have allowed (a fact soon to be fully appreciated) for a solution without violating spherical symmetry.

The fact that a real scalar is involved has here a deep theoretical consequence. As is well known, based on renormalizability arguments, the bare scalar potential in $d$-dimensions can be at most of order $\frac{2 d}{d-2}$. With this in mind, we are after the exciting possibility that a $6^{\text {th }}$-order potential plays on our 3 -dimensional world-manifold a role similar to the one played by the $\left(4^{\text {th }}\right.$-order $)$ Higgs potential in the background spacetime. Now comes a simple observation: for a real $\Phi$ we may have, as advertised at most 3 independent isolated minima. For a complex $\Phi$, however, in the absence of odd self-interaction terms, one $U(1)_{\mathrm{g}}$-degenerate minimum may be solely accompanied by a trivial minimum at the origin. Denoting the three solutions of $\frac{\partial V}{\partial \Phi}=0$ by $\langle\Phi\rangle_{i}=\Lambda_{i}$, each family is characterized by a different spontaneously induced surface-tension $\Lambda_{i}$. This in turn gets directly translated into the Dirac language [3] of different radii and masses. For example,

$$
r_{i}=\frac{3 q^{2}}{4 \Lambda_{i}} \quad, \quad M_{i}=\frac{9 q^{2}}{4 r_{i}} \quad \text { in the } m \rightarrow \infty \text { Dirac limit. }
$$

Also note that, reflecting the polynomial structure of the potential, the individual 'lepton numbers' are preserved up to exponentially suppressed tunneling effects.

\section{6. $\mathrm{SU}(2)_{\mathrm{spin}} \otimes \mathrm{U}(1)_{\mathrm{g}}$ Group Theory}

A magnetic monopole-like configuration is known to have some dramatic geometrical consequences [12], in particular if it lives on the $S^{2}$ sphere. To analyze the group theoretical spectrum of the allowed harmonic excitations, consider a generic secondary-charged world-manifold scalar field $\psi\left(y^{\alpha}\right)$ whose covariant derivative (with respect to the curved geometry as well as the $U(1)_{\mathrm{g}}$ gauge group) is given by 


$$
D_{\alpha} \psi=\psi_{; \alpha}+i e a_{\alpha} \psi
$$

The corresponding Laplacian is explicitly given by

$$
g^{\alpha \beta} D_{\alpha} D_{\beta} \psi=\left(-\frac{\partial^{2}}{\partial \tau^{2}}+\frac{1}{r^{2} \sin ^{2} \theta} \cdot \frac{\partial}{\partial \theta}\left(\sin \theta \frac{\partial}{\partial \theta}\right)+\frac{1}{r^{2} \sin ^{2} \theta}\left(\frac{\partial}{\partial \phi}+i e g(1-\cos \theta)\right)^{2}\right) \psi .
$$

Up to the additive term $\frac{e^{2} \mathrm{~g}^{2}}{r^{2}}$, the angular part of the Laplacian is recognized as the Casimir operator $J^{2}=J_{1}^{2}+J_{2}^{2}+J_{3}^{2}$ of spatial $S U(2)$. Indeed, the three generators are

$$
\begin{aligned}
& J_{1}=i \sin \phi \frac{\partial}{\partial \theta}+\cos \phi\left(i \frac{\cos \theta}{\sin \theta} \cdot \frac{\partial}{\partial \phi}+\frac{e \mathrm{~g}}{\sin \theta}(1-\cos \theta)\right), \\
& J_{2}=-i \cos \phi \frac{\partial}{\partial \theta}+\sin \phi\left(i \frac{\cos \theta}{\sin \theta} \cdot \frac{\partial}{\partial \phi}+\frac{e \mathrm{~g}}{\sin \theta}(1-\cos \theta)\right), \\
& J_{3}=-i \frac{\partial}{\partial \phi}+e \mathrm{~g} .
\end{aligned}
$$

An eigenstate of $J_{3}$, namely, $J_{3} \psi=s \psi$, which happens to be the highest weight in its representation, that is $J^{+} \psi=0$, is found to be

$$
\psi \approx\left(\sin \frac{\theta}{2}\right)^{s-e g}\left(\cos \frac{\theta}{2}\right)^{s+e g} e^{i(s-e g) \phi} .
$$

The lower states of the associated representation can be constructed by successive $J^{-}$ operations, with the characteristic effect of decreasing the powers of $\left(\sin \frac{\theta}{2}\right)$ and/or $\left(\cos \frac{\theta}{2}\right)$ by units of 1 at each step. This tells us that the representation is non-singular if $s \pm e \mathrm{~g}$ is non-negative, and is furthermore finite provided

$$
s \pm e \mathrm{~g}=\text { non-negative integer } .
$$

This completes the classification of the allowed $S U(2)_{\operatorname{spin}} \otimes U(1)_{\mathrm{g}}$ representations. In particular, a spin -0 field can only tolerate $e g=0$. This constitutes our previous insight regarding the reality of the scalar field $\Phi$ invoked to resolve the family puzzle. More interesting, however, and soon to be put to work, are the two independent spin $-1 / 2$ representations associated with $e \mathrm{~g}=+\frac{1}{2}$ and $e \mathrm{~g}=-\frac{1}{2}$, respectively. They forcefully, demonstrate how a half-integer spin can spontaneously arise from a sophisticated apparently scalar configuration. This highly reminds us of the Skyrme soliton [13], where a lump-like solution of a classical scalar field is quantized as a fermion. We find it ironic that the famous Dirac quantization condition $e \mathrm{~g}=\frac{n}{2}$ gets realizes here for the secondary $U(1)_{\mathrm{g}}$ and not, as was originally meant [10], for the parent $U(1)_{\text {e.m. }}$. At this point, it is worth recalling once again that $g$ also serves as the bubble's electric charge $q$. 


\section{A Pedagogical No-Go Theorem}

Picking up one of the two spin-1/2 representations, let us study the role of an additional term:

$$
\bar{S}^{(4)}=\oint \sqrt{-\operatorname{det} g} \sum_{i=\downarrow, \uparrow} g^{\alpha \beta}\left(D_{\alpha} \psi_{i}\right)^{\dagger}\left(D_{\beta} \psi_{i}\right) d^{3} y
$$

where $D_{\alpha} \psi_{i}=\left(\partial_{\alpha}+i e a_{\alpha}\right) \psi_{i}$. A scalar potential $W\left(\psi^{\dagger} \psi\right)$ is optional, but will distract us from the main stream. As far as the variation with respect to $\psi_{i}$ is concerned, two solutions are possible:

$$
\begin{aligned}
& \mathrm{g}=+\frac{1}{2 e} \quad \Rightarrow \quad\left\{\begin{array}{l}
\psi_{\uparrow}=a \cos \frac{\theta}{2}, \\
\psi_{\downarrow}=b \sin \frac{\theta}{2} e^{-i \phi},
\end{array}\right. \\
& \mathrm{g}=-\frac{1}{2 e} \quad \Rightarrow \quad\left\{\begin{array}{l}
\psi_{\uparrow}=c \sin \frac{\theta}{2} e^{i \phi}, \\
\psi_{\downarrow}=-d \cos \frac{\theta}{2} .
\end{array}\right.
\end{aligned}
$$

The good news are that the constant of integration $g$ is no longer arbitrary. Namely, it may only take the specific values $\pm \frac{1}{2 e}$, corresponding to fixed (opposite) electric charges. We note in passing that the fractional quark charges are expected to arise naturally once $U(1)_{\text {e.m. }}$. is embedded within a grand unifying theory. A unified generalization of the present idea probably involves a secondary 't Hooft-Polyakov monopole [14].

The above solution may give us the false impression that two scalar components suffice to describe an electron (or a positron). The trouble is simple: the above is not a self-consistent solution. To see the point, concentrate on $\frac{\delta L}{\delta a_{\alpha}}$ which receives here its only non-trivial contribution. Self-consistency then takes the covariant current conservation form:

$$
\left(\psi^{\dagger} \partial_{\alpha} \psi-\left(\partial_{\alpha} \psi^{\dagger}\right) \psi\right)+2 i e a_{\alpha} \psi^{\dagger} \psi=0
$$

which is automatically satisfied for $\alpha=\tau, \theta$, but fails to hold for $\alpha=\phi$, unless

$$
a^{2}=b^{2} \quad, \quad c^{2}=d^{2},
$$

but this is sick from the $S U(2)_{\text {spin }}$ point of view (e.g. $J^{ \pm} \psi$ takes us off the representation). However, such a spontaneous symmetry violation comes with no surprise. Having in mind that the Dirac algebra does not have a $2 \times 2$ Pauli realization (The $\gamma^{\mu}$-matrices are $4 \times 4$ in their minimal representation), one could have expected some kind of no-go situation. Indeed, the remedy relies upon constructive co-operation between the two independent spin-1/2 representations. 


\section{The Electron (Positron) Configuration}

Taking into account both available spin-1/2 representations, $\bar{S}^{(4)}$ is replaced by the charge-conjugation invariant $(e \rightarrow-e)$ kinetic term

$$
S^{(4)}=\oint \sqrt{-\operatorname{det} g}\left(\sum_{\substack{i=+,-j=\uparrow, \downarrow}} g^{\alpha \beta}\left(D_{\alpha} \psi_{i j}\right)^{\dagger}\left(D_{\beta} \psi_{i j}\right)+W\left(\psi^{\dagger} \psi\right)\right) d^{3} y
$$

where $D_{\alpha} \psi_{ \pm}=\left(\partial_{\alpha} \pm i e a_{\alpha}\right) \psi_{ \pm}$. The corresponding self-consistency condition takes the generalized form

$$
\left(\psi_{+}^{\dagger} \partial_{\alpha} \psi_{+}-\left(\partial_{\alpha} \psi_{+}^{\dagger}\right) \psi_{+}\right)-\left(\psi_{-}^{\dagger} \partial_{\alpha} \psi_{-}-\left(\partial_{\alpha} \psi_{-}^{\dagger}\right) \psi_{-}\right)+2 i e a_{\alpha}\left(\psi_{+}^{\dagger} \psi_{+}+\psi_{-}^{\dagger} \psi_{-}\right)=0 .
$$

At this stage, our discussion bifurcates. First we present the electron configuration $\left(\mathrm{g}=+\frac{1}{2 e}\right)$, and then go on to consider its positron $\left(\mathrm{g}=-\frac{1}{2 e}\right)$ companion.

The electron: For $\mathrm{g}=+\frac{1}{2 e}$, we derive

subject to

$$
\Psi=\left\{\begin{array}{l}
\psi_{+\uparrow}=a \cos \frac{\theta}{2} \\
\psi_{+\downarrow}=b \sin \frac{\theta}{2} e^{-i \phi} \\
\psi_{-\uparrow}=c \sin \frac{\theta}{2} e^{i \phi} \\
\psi_{-\downarrow}=-d \cos \frac{\theta}{2}
\end{array}\right.
$$

by virtue of the above self-consistency condition. Dismissing the two redundant solutions $(a=b=0$, and $c=d=0)$ discussed earlier, we can finally identify the polarized electron configurations

$$
\Psi_{\uparrow}=\left\{\begin{array}{l}
\psi_{+\uparrow}=\mathrm{A} \cos \frac{\theta}{2} \\
\psi_{+\downarrow}=0 \\
\psi_{-\uparrow}=\mathrm{A} \sin \frac{\theta}{2} e^{i \phi} \\
\psi_{-\downarrow}=0
\end{array} \quad, \quad \psi_{\downarrow}=\left\{\begin{array}{l}
\psi_{+\uparrow}=0 \\
\psi_{+\downarrow}=\mathrm{A} \sin \frac{\theta}{2} e^{-i \phi} \\
\psi_{-\uparrow}=0 \\
\psi_{-\downarrow}=-\mathrm{A} \cos \frac{\theta}{2}
\end{array}\right.\right.
$$

The underlying spherical symmetry is expressed by the fact that, for each configuration, $\Psi^{\dagger} \Psi$ is independent of $\phi, \theta$. The role of the scalar potential $W\left(\Psi^{\dagger} \Psi\right)$ in fixing $\mathrm{A}(r, t)$ is to be clarified soon. As we shall see, its presence is absolutely necessary for having a static ( $t$-independent) solution. The $6^{t h}$-order polynomial structure of $W\left(\Psi^{\dagger} \Psi\right)$ and the complexity of $\Psi$ indicate that A can take at most one non-vanishing value. The total number of (three) families is not expected to increase by the introduction of $\psi_{i j}$.

The Positron: For $\mathrm{g}=-\frac{1}{2 e}$, interchanging the roles played by $\psi_{+}$and $\psi_{-}$, a similar calculation reveals 


$$
\Psi^{c}=\left\{\begin{array}{l}
\psi_{+\uparrow}=a \sin \frac{\theta}{2} e^{i \phi} \\
\psi_{+\downarrow}=-b \cos \frac{\theta}{2} \\
\psi_{-\uparrow}=c \cos \frac{\theta}{2} \\
\psi_{-\downarrow}=d \sin \frac{\theta}{2} e^{-i \phi}
\end{array}\right.
$$

again with $a^{2}+d^{2}=b^{2}+c^{2}$. The physical polarized positron configurations are the following

$$
\Psi_{\uparrow}^{c}=\left\{\begin{array}{l}
\psi_{+\uparrow}=\mathrm{A} \sin \frac{\theta}{2} e^{i \phi} \\
\psi_{+\downarrow}=0 \\
\psi_{-\uparrow}=\mathrm{A} \cos \frac{\theta}{2} \\
\psi_{-\downarrow}=0
\end{array} \quad, \quad \Psi_{\downarrow}^{c}=\left\{\begin{array}{l}
\psi_{+\uparrow}=0 \\
\psi_{+\downarrow}=-\mathrm{A} \cos \frac{\theta}{2} \\
\psi_{-\uparrow}=0 \\
\psi_{-\downarrow}=\mathrm{A} \sin \frac{\theta}{2} e^{-i \phi}
\end{array} .\right.\right.
$$

\section{Light Mass?}

Recalling that the full action $S^{(1)}+S^{(2)}+S^{(3)}+S^{(4)}$ exhibits no explicit $t\left(y^{\alpha}\right)$ dependence, the total energy of the system is clearly conserved. To be somewhat more specific, with spherical symmetry imposed, we have

$$
\frac{\partial}{\partial \tau}\left(\frac{\delta L}{\delta t, \tau}+\frac{q^{2}}{2 r}\right)=0,
$$

where the $\frac{q^{2}}{2 r}$ piece is the Dirac contribution of the surrounding electromagnetic field. The $t,_{\alpha}$-dependence of the Lagrangian is hidden within $\frac{\delta g_{\tau \tau}}{\delta t,,_{\tau}}=-2, \frac{\delta A_{\tau}}{\delta t_{,}}=\frac{q}{r}$. The general analysis of the total energy lies beyond the scope of the present paper. Here, our only intention is to demonstrate the existence of stable static configurations. We minimize $E(r, \Phi, \mathrm{A})$, and speculate about the lightness of the mass.

It takes some algebra to arrive at the energy formula

$$
E(r, \Phi, \mathrm{A})=\left(V(\Phi)+W\left(\mathrm{~A}^{2}\right)\right) r^{2}+\frac{1}{2} \mathrm{~A}^{2}+\frac{\mathrm{g}^{2}}{2 m r^{2}}+\frac{3 q^{2}}{2 r},
$$

where $W_{\text {eff }} \equiv W+\frac{\mathrm{A}^{2}}{2 r^{2}}$ is the fermionic contribution to the surface tension . $V(\Phi)+$ $W\left(\mathrm{~A}^{2}\right)$ can be harmlessly embedded within a more general potential $U\left(\Phi, \mathrm{A}^{2}\right)$. Noticeably, a major Dirac drawback is still present. For radii smaller than $10^{-15} \mathrm{~cm}$, one obtains masses heavier than $100 m_{e}$. Is there a physical way to make the mass lighter, or perhaps arbitrarily light? To answer this question, we recall that the 
previously mentioned curvature option has not yet been fully exploited. The bubble curvature may enter either via a conformal coupling $-\frac{1}{8} \oint R \Phi^{2} \sqrt{-\operatorname{det} g} d^{3} y$, which gives $\Delta E=+\frac{1}{8} \Phi^{2}$, and/or via 'surface gravity':

$$
S^{(5)}=\tilde{m} \oint R \sqrt{-\operatorname{det} g} d^{3} y,
$$

whose tenable contribution $\Delta E=-2 \tilde{m}$ is of-course most welcome. This may be another indication that an Einstein-Maxwell generalization is mandatory, with the self-consistent mass $M$ parametrizing the surrounding Reissner-Nordstrom geometry. At any rate, the overall situation reminds us of the standard electroweak model, where the origin of mass is well established yet nothing specific can be said about individual fermionic masses.

\section{Summary}

Invoking world-manifold gauge field theory, we have revived the Dirac idea of a finite size electron, spin and family included. Our final product is a threefold family of electrically charged spin-1/2 static Dirac bubbles. The main result, formulated by eqs. (18), (20), establishes a one-to-one correspondence between four world-manifold scalar configurations and the four components of the Dirac spinor. On the technical side, a key role is played by the spherically symmetric magnetic monopole groundstate configuration of the secondary $U(1)_{\mathrm{g}}$ gauge field. Namely, it provides the locally conserved charge distribution for the primary $U(1)_{\text {e.m. }}$ electric monopole, and furthermore allows (in a group theoretical manner) for the spontaneous generation of spin. The strength of the magnetic monopole, also serving as the bubble's electric charge, is uniquely fixed by the Dirac quantization condition. The family puzzle is resolved by minimizing a ( $6^{\text {th }}$-order) surface-tension potential; at most three families are expected. As far as the Fermi mass problem is concerned, no quantitative progress can be reported at the present stage. The situation may change, however, once a better understanding of the so-called surface gravity becomes available. The complement list of topics to be discussed in future includes the interactions with external sources, the non-Abelian generalization (focusing on $S U(3)_{\text {color }} \otimes U(1)_{\text {e.m. }}$.), the self-consistent gravitational treatment, and the provocative massless limit. 


\section{References}

[1] M. Abraham, Phys. Z. 4 (1902) 57;

H.A. Lorentz, Proc. Acad. Amst. 6 (1904) 809.

[2] A. Einstein, Sitz. Preu. Acad. d. Wiss. (1919).

[3] P.A.M. Dirac, Proc. Roy. Soc. A268 (1962) 57.

[4] J.C. Pati, A. Salam and J. Strathdee, Phys. Lett. 59B (1975) 265;

H. Harari, Phys. Lett. 86B (1979) 83;

M.A. Shupe, Phys. Lett. 86B (1979) 78;

G. 't Hooft, in "Recent Developments in Gauge Theories", ed. G. "t Hooft, (Plenum, N.Y., 1980).

[5] H. Harari and N. Seiberg, Phys. Lett. 98B (1981) 269;

O.W. Greenberg and J. Sucher, Phys. Lett. 99B (1981) 339;

J. Ellis, M.K. Gaillard, L. Maiani and B. Zumino, Phys. Lett. 94B (1980) 343;

A. Davidson and J. Sonnenshein, Phys. Rev. Lett. 50 (1983) 1339.

[6] F. Wilczek, Phys. Rev. Lett. 49 (1982) 957;

F. Wilczek and A. Zee, Phys. Rev. Lett. 51 (1983) 2250.

[7] A. Davidson and U. Paz, (in preperation).

[8] A. Davidson and K.C. Wali, Nucl. Phys. B349 (1991) 581.

[9] A. Davidson and E.I. Guendelman, Phys. Lett. 251B (1990) 250.

[10] P.A.M. Dirac, Proc. Roy. Soc. 133 (1931) 60;

J. Schwinger, Science 165 (1969) 757;

R. Jackiw and C. Rebbi, Phys. Rev. Lett. 36 (1976) 1116.

[11] Y. Nambu, Phys. Rev. D4 (1971) 1193;

A.P. Balachandran, A. Stern and B.S. Skagerstam, Phys. Rev. D20 (1979) 439;

N.K. Nielsen, Nucl. Phys. B167 (1980) 249;

E. Witten, Nucl. Phys. B249 (1985) 557;

A. Vilenkin and T. Vachaspati, Phys. Rev. Lett. 58 (1987) 1041;

B. Carter, Phys. Lett. 224B (1989) 61.

[12] S. Randjbar-Daemi, A. Salam and J. Strathdee, Nucl. Phys. B214 (1983) 491.

[13] T.H.R. Skyrme, Proc. Roy. Soc. A260 (1961) 127;

A.P. Balachandran, G. Marmo, B.S. Skagerstam and A. Stern, Nucl. Phys. B162 (1980) 385; 
E. Witten, Nucl. Phys. B223 (1983) 422;

M.V. Berry, Proc. Roy. Soc. A392 (1984) 45.

[14] G. 't Hooft, Nucl. Phys. B79 (1974) 276;

A.M. Polyakov, JETP Lett. 20 (1974) 194. 